\title{
Alexia and agraphia in Wernicke's aphasia
}

\author{
HOWARD S KIRSHNER, WANDA G WEBB
}

From the Departments of Neurology and Hearing and Speech Science, Vanderbilt University School of Medicine, Nashville, Tennessee, USA

SUMMARY Three patients with otherwise typical Wernicke's aphasia showed consistently greater impairment of reading than auditory comprehension. While this syndrome resembles alexia with agraphia, the paraphasia of speech, repetition, and naming underline the aphasic nature of the disorder. Together with previous reports of isolated word deafness in Wernicke's aphasia, these cases suggest a relative independence of auditory and visual language processing.

Wernicke's aphasia is traditionally characterised ${ }^{12}$ as a disorder of central language processes, affecting the different modalities of language input and output more or less equally. Thus reading is impaired along with auditory comprehension, and writing, like spontaneous speech, is marred by paraphasic substitutions. Anomia and paraphasic substitutions in naming and repetition complete the picture. The equivalence of deficits in reading and auditory comprehension is often explained by the concept that reading is learned by transcoding of visual language stimuli to previously acquired auditory word associations, stored in Wernicke's area. ${ }^{3}$ Disruption of the auditory association area would thus interfere not only with auditory comprehension, but also with reading.

In a previous communication, ${ }^{4}$ we discussed cases of Wernicke's aphasia with spared reading ability and compared this auditory-predominant sub-type of Wernicke's aphasia to the traditional syndrome of pure word deafness. The present report concerns three cases of Wernicke's aphasia, all secondary to ischaemic stroke, in which reading was more impaired than auditory comprehension, both acutely and after partial recovery. Whereas the auditorypredominant cases of Wernicke's aphasia resemble pure word deafness, this second, visual-predominant sub-type resembles the classical syndrome of pure alexia with agraphia, an acquired illiteracy in which reading and writing are impaired in the absence of other language deficits. The paraphasic productions of our patients in spontaneous speech, naming,

Address for reprint requests: Howard S Kirshner, MD, Department of Neurology, Vanderbilt University School of Medicine, Nashville, Tennessee 37232, USA.

Received 21 November 1981 and in revised form 6 March 1982. Accepted 18 March 1982 repetition, and oral reading, however, distinguish their deficit from pure alexia without agraphia and link them more closely to Wernicke's aphasia. These cases add to previous evidence that the auditory and visual modalities of language may be affected to different degrees in Wernicke's aphasia and suggest at least a partial independence of auditory and visual language processing.

\section{Methods and report of cases}

Three aphasic patients, all victims of acute left hemisphere strokes, were studied within five days after onset and again four to six weeks later. All had at least a partial high school education and good premorbid reading skills. The tests employed are the same as those described previously.

Case 1 This 67-year-old right-handed male, in good health except for hypertension and prior myocardial infarction, entered the hospital after an episode of blurred vision, lightheadedness, nausea, and left-sided weakness. Initial examination disclosed only a left homonymous hemianopsia and left hemisensory deficit, both of which resolved over the next few hours. A computed tomographic (CT) scan was normal. Three days later, during cerebral arteriography, the patient became restless and "confused". The arteriogram showed nonstenosing plaque formation at the right carotid bifurcation; the left carotid bifurcation and intracranial vessels were unremarkable. On examination the patient repetitively uttered phrases such as "let me out, ... . let me get over". He followed no commands, called his daughter "Mama", and made many paraphasic errors. He had a dense right homonymous hemianopsia but no apparent motor or sensory deficits. By the next morning he followed some simple commands but not multistep directions. He manifested almost total anomia for objects and colours.

The patient improved steadily over the next few days. Five days after onset he named eight of ten pictures, five of six colours, and eight of ten small letters. He correctly followed seven of eight commands of increasing complexity, either spoken or presented in printed form. On a 
matching-to-sample test battery modified from Sidman $e t$ $a l^{5}$ he performed with greater than $90 \%$ accuracy in matching single, dictated or printed small letters to printed capital letter choices and in matching single words presented in printed, dictated, or dictated, spelled form to printed word choices. He read aloud 10/10 3-letter words. On the Boston Diagnostic Aphasia Examination (BDAE), ${ }^{6}$ performed four days after onset, the patient's oral expression was fluent but marked by both literal and verbal paraphasias and word-finding difficulty. Confrontation naming showed prolonged latencies and some errors on pictures, body parts, and colours. Auditory comprehension subtests showed normal responses to multistep commands and complex ideational material. Repetition was marked by paraphasic substitutions, especially on low probability phrases. On reading tests he was unable to comprehend passages of more than two sentences, and oral reading was slow, effortful, and contaminated by paralexic errors. He could write short sentences to dictation but made spelling errors on longer words. Re-examination with the BDAE four weeks after onset showed improvement in all areas, but with continued paraphasic errors in spontaneous speech, repetition, and oral reading. His most significant disability remained that of alexia, with inability to comprehend paragraph-length material.

The Token Test' ${ }^{7}$ was performed four weeks after onset, both in the standard, auditory form and with the instructions presented in printed form. He correctly performed $94 \%$ of the auditory and $82 \%$ of the visual instructions (by weighted scores).

A repeat CT scan (fig 1), performed 6 days after onset, showed an area of low density involving predominantly the left angular gyrus, with extension into the supramarginal gyrus, posterior-most superior temporal gyrus, and inferior-most superior parietal lobule, along with subjacent white matter. ${ }^{8}$ The patient continued to improve in speech and language, with mild paraphasia of spontaneous speech and continued reading difficulty when last seen three months after onset.

Case 2 This 69-year-old hypertensive, diabetic righthanded lady presented with several episodes of left monocular blurred vision and one of transient left hemiparesis associated with "slurred" speech. Examination revealed right carotid and left supraclavicular bruits. No fixed neurologic deficits were found. CT scan of the head was normal. Cerebral arteriography revealed tight stenosis of the right internal carotid artery, severe plaque formation and $50-60 \%$ stenosis of the left internal carotid artery, and stenosis of the proximal left vertebral and subclavian arteries. Following the arteriogram the patient was noted to be "confused"; on examination, her speech was rambling, with numerous spontaneous paraphasic errors and neologisms. She did not respond to commands or yes/no questions. She showed no apparent appreciation of visual stimuli and failed to count fingers or blink to visual threat. Spontaneous movements and withdrawal to pinprick were strong and symmetric in all extremities. Reflexes were symmetic, with flexor plantar responses. On the following day she remained confused but reached out to grasp objects with either hand, more consistently in the left field of vision. She continued to speak paraphasically but expressed some appropriate phrases, including a complaint of headache. She failed to identify objects or colours. By the fourth day she looked about the room, recognised family members, and spoke in full sentences, though with many word substitutions. Over the next few days she regained the ability to name some objects but not uncommon objects or parts of objects. Her auditory comprehension

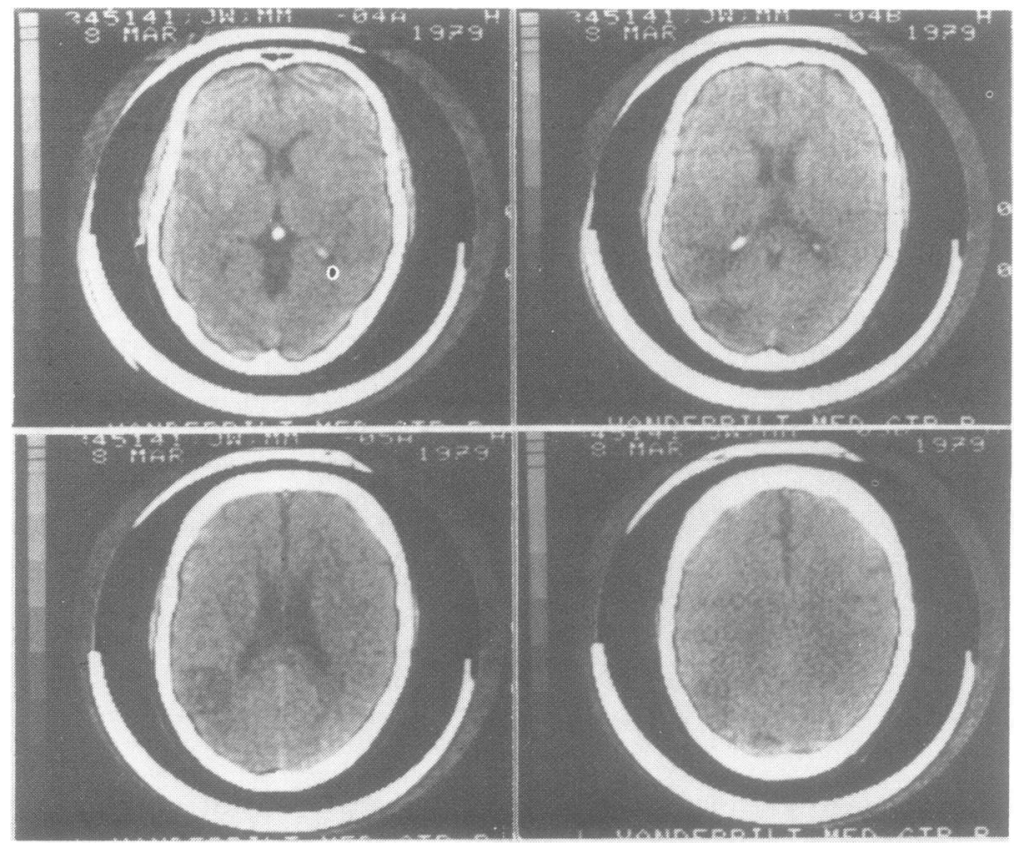

Fig 1 Computed tomographic (CT scan), Case 1, showing an area of reduced attenuation, consistent with infarction in the region of the left angular gyrus. 

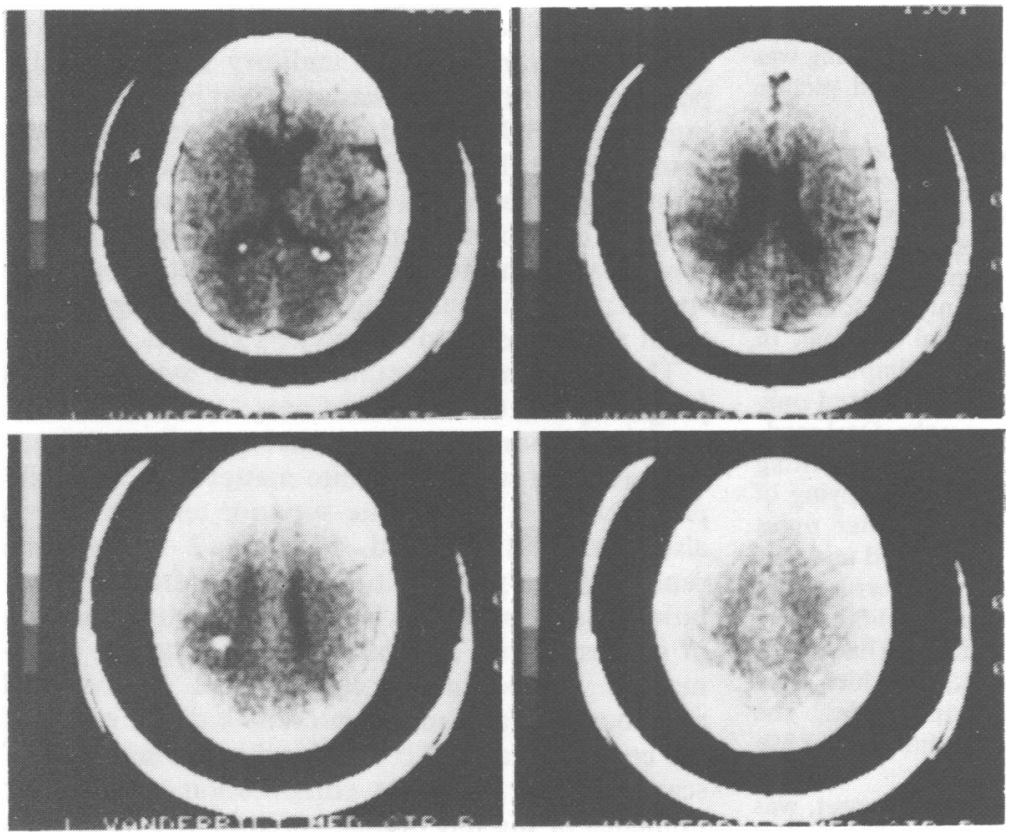

Fig 2 CT scan, Case 2, showing infarction in the vicinity of the left angular gyrus. The small area of increased density represents haemorrhagic infarction.

and following of commands recovered substantially, except for left-right errors. In contrast, she remained almost entirely unable to read or write. She could not read more than single words aloud, follow the simplest printed commands, or sign her name. She was unable to perform even simple calculations, such as " $7+5$ ", to copy or construct geometrical figures, or to identify left and right. She was able to point to specific fingers on her own and the examiner's hand.

Speech and language evaluations were carried out 2-3 weeks after onset. She named $16 / 20$ capital letters, 17/20 pictures, and read aloud 19/20 picture names. In contrast, she could not write the names of any of ten pictures. On the matching-to-sample battery, she correctly matched $19 / 20$ dictated and 8/10 printed small letters to the correct capital letters. She also matched $20 / 20$ printed, $20 / 20$ dictated, and 19/20 dictated, spelled words to pictures. Given a series of word lists differing in length and frequency of occurrence, she read aloud the frequent words accurately, whether short or long, but she made frequent errors on the unfamiliar words and was totally unable to pronounce nonsense trigrams. At the time of retesting six weeks after onset, the patient named $20 / 20$ pictures orally and $10 / 20$ in writing. On the BDAE, spontaneous speech was fluent, with frequent literal paraphasic errors. The patient exhibited moderate dysnomia for objects, actions, colours, numbers, and body parts. Auditory comprehension was impaired only for multistep commands and complex ideational material. Single word and sentence repetition showed literal paraphasic errors. Oral reading was severely impaired, with marked hesitancy and both literal and verbal paraphasic errors. She failed to read sentences or paragraphs for meaning and made occasional errors even in single word recognition. Writing ability was limited to the copying of single letters and numbers; she failed to write letters, numbers, or words to dictation. Retesting six weeks after onset revealed almost normal auditory comprehension and mild, persistent dysnomia and paraphasic speech. Reading and writing, though improving, remained severely deficient. The Token Test, performed three weeks after onset, showed $90 \%$ correct for auditory and $39 \%$ correct for visual instructions.

A second CT scan, 18 days after onset, revealed a low density area involving the left angular gyrus and subjacent white matter, with slight extension into the supramarginal gyrus and superior parietal lobule. The entire temporal lobe appeared spared. ${ }^{8}$ The patient's subsequent course was one of gradual improvement, with mild paraphasic speech and word-finding difficulty, but persistent and frustrating impairments in reading and writing.

Case 3 This 60-year-old right-handed male was previously in good health except for hypertension, amputation of the right leg, and a progressive left ear hearing loss. Audiometry disclosed a moderate conductive hearing loss on the left, with only high frequency loss on the right. While hospitalised for evaluation of the hearing loss he suddenly developed a right hemiparesis and aphasia, with initial descriptions of his speech as "slurred" and "nonsensical". By day 5 he manifested a clearcut fluent aphasia, while the hemiparesis had improved to mild weakness of the right face and arm. Visual fields were intact. Bedside aphasia testing six days after onset revealed fluent speech with word-searching pauses, circumlocutions, and frequent literal and verbal paraphasic errors. He named $8 / 8$ objects, $3 / 3$ parts of objects, and $10 / 10$ pictures. He repeated simple and complex phrases with few errors. Despite the hearing loss he followed 8/8 spoken commands of increasing complexity and answered appropriately to yes/no and nonsense questions. In contrast, he was able to carry out or read aloud only $5 / 8$ printed commands. Writing was 
limited to his signature and single, dictated words; he could not write even short sentences to dictation. Matching was nearly errorless for the single letter, word, and picture items.

Ōn the BDAE, performed on days 5-7, spontaneous speech was characterised by fluent phraseology with many clichés, few meaningful nouns, paraphasic errors, and some neologistic jargon. Naming was mildly impaired, and repetition was accurate but contaminated by paraphasic errors. Auditory comprehension was impaired only on four and five part commands and complex ideational material. In contrast, reading comprehension was severely impaired for more than single word items. He correctly answered only $20 \%$ of questions on sentences and paragraphs. Oral reading of sentences contained many paraphasic errors. Writing was poor even for dictated single words and copying of words and sentences. Retesting five weeks after onset showed improvement in spontaneous speech and auditory comprehension, with persistent paraphasic errors and word-finding difficulty. Reading of sentences, both aloud and for comprehension, remained impaired. The Token Test was performed 16 days after onset, by which time comprehension had improved in both modalities. The patient correctly carried out $93 \%$ of the auditory and $88 \%$ of the visual instructions.

A CT scan, performed within five hours of onset, was normal. A repeat study was not obtained. The clinical course was one of substantial improvement in all aspects of language. Only mildly paraphasic speech and reading difficulty remained as clinically evident deficits.

\section{Discussion}

Since its original description in $1874,{ }^{9}$ Wernicke's aphasia has been considered a disorder of central language processes, independent of specific language modalities. Disruption of the auditory word association area in the superior temporal gyrus (Wernicke's area) interferes with auditory processing. Reading, by the traditional formulation, ${ }^{39}$ is learned by the transcoding of visual language stimuli to previously learned auditory word associations; alexia would thus also be expected with lesions in this region.

In a previous communication, ${ }^{4}$ we discussed cases of Wernicke's aphasia with poor auditory comprehension but relatively spared reading. This disorder resembles the syndrome of pure word deafness, except for the paraphasic speech, repetition, and naming. The preserved reading ability in these cases appears to reside in the left parietal and occipital lobes, ${ }^{410}$ or possibly even in the right hemisphere. ${ }^{11}$

The three cases discussed here suggest a second modality-specific deficit pattern in Wernicke's aphasia, that of greater impairment of reading than auditory comprehension. All three patients had completed at least ten years of school and read fluently prior to their strokes. The greater involvement of reading than of auditory comprehension persisted over time and was apparent in bedside testing, on the BDAE, and on the auditory and visual forms of the Token Test. Cases 2 and 3 showed significant agraphia as well as alexia, suggesting a primary diagnosis of pure alexia with agraphia. All three cases, however, had persistent paraphasic speech, repetition, naming, and oral reading, linking them more closely to Wernicke's aphasia.

The anatomic substrate of this syndrome, as judged by the CT scans of Cases 1 and 2, involved the left inferior parietal region, especially the angular gyrus and subjacent white matter. In Case 1 the posterior-most part of the superior temporal gyrus also appeared involved. In Case 2 the aphasia remained as a persistent focal deficit after the resolution of a more diffuse postarteriography syndrome of confusion and cortical blindness. Cortical blindness is a recognised complication of cerebral arteriography and may represent a direct effect of the contrast material. ${ }^{12}$ The focal lesion seen on CT scan, however, suggests embolisation to posterior branches of the left middle cerebral artery, as does the sudden onset of the language disorder in all three cases.

While Wernicke himself assigned the auditory word association area to the posterior part of the superior temporal gyrus, ${ }^{9}$ subsequent aphasiologists have differed in its localisation. The inferior parietal lobule, or supramarginal and angular gyri, has been included by some authors. ${ }^{13}$ Paraphasic speech and impaired auditory comprehension are thus not unexpected with lesions in this vicinity. The greater involvement of reading than of auditory comprehension, however, cannot be explained by involvement of the auditory association area alone, but rather must reflect the importance of the angular gyrus region to reading.

Dejerine, ${ }^{14}$ in 1892 , described the syndrome of pure alexia with agraphia in a patient with an infarct of the left angular gyrus. He believed the angular gyrus to be a centre for visual word images, analogous to the superior temporal gyrus as a centre for auditory word images. Wernicke, ${ }^{9}$ and more recently Geschwind, ${ }^{15}$ emphasised the role of the angular gyrus in cross-modal sensory associations. Instead of removing a cortical word-image centre, a lesion of the angular gyrus would disrupt reading by disconnecting visual to auditory transcoding. By either explanation, an angular gyrus lesion would produce alexia without affecting either auditory comprehension or speech production. Dejerine ${ }^{14}$ stated that alexia and agraphia could either accompany a Wernicke's aphasia or exist in pure form. Some recent authors ${ }^{21617}$ have attempted to distin- 
guish alexia with agraphia from "aphasic alexia", or the alexia associated with Wernicke's aphasia. On the other hand, both Kertesz ${ }^{17}$ and Brain ${ }^{18}$ noted that alexia with agraphia can remain as a residual deficit after recovery from Wernicke's aphasia. Benson $^{19}$ stated that only in rare instances does alexia with agraphia occur without any signs of aphasia.

Modern classifications of alexia have emphasised three principal groups: alexia with agraphia, alexia without agraphia, and alexia associated with aphasia. ${ }^{3}$ Alexia with agraphia has already been discussed. Alexia without agraphia is an easily separable syndrome seen with lesions of the left occipital lobe, often including the splenium of the corpus callosum. Dejerine ${ }^{14}$ explained this syndrome by a disconnection of visual information perceived by the intact right occipital lobe from the left angular gyrus. Aphasic alexia has included different groups of aphasic cases. Benson ${ }^{19}$ has called attention to alexia in Broca's aphasia, under the term "third alexia". The nonfluent speech of these patients, of course, should distinguish them readily from the other alexic groups. Finally, the alexia of Wernicke's aphasia may be considered as a separate, fourth group, or it may be combined with the first syndrome, alexia with agraphia. Benson ${ }^{19}$ has suggested the terms "central alexia" for the alexia with agraphia cases, "posterior alexia" for alexia without agraphia, and "anterior alexia" for the reading disorder seen with Broca's aphasia.

An alternative explanation for the findings of fluent aphasia and alexia in our patients would be a combination of conduction aphasia and alexia with agraphia. Conduction aphasia, a syndrome in which paraphasic speech and impaired repetition occur in the presence of intact auditory comprehension, is associated with lesions of either the left supramarginal gyrus or the left superior temporal gyrus. ${ }^{20}$ Factors against the diagnosis of conduction aphasia in our patients include the presence at least initially of auditory comprehension deficit, the absence of a severe repetition disturbance out of proportion to other language deficits, and the absence of disproportionate literal as compared to verbal paraphasic errors. For these reasons we prefer the diagnosis of Wernicke's aphasia with alexia. The most important point, however, is the linkage of alexia with agraphia to the fluent aphasias.

Numerous studies have attempted to distinguish alexic syndromes by their relative impairments of word versus letter reading. In alexia without agraphia, preservation of individual letter reading has been amply documented. ${ }^{21-24}$ The alexia of Broca's aphasia, on the other hand, produces a major deficit in the naming and reading of single letters, even when some words can be read in a global manner. ${ }^{192526}$ In Wernicke's aphasia, single letters may be processed well, as seen in our cases, and difficulty increases with increasing complexity of words and phrases. ${ }^{16}$ In alexia with agraphia, some authors $^{27}$ have found letter greater than word alexia, much like that seen in Broca's aphasia. Other authors, ${ }^{16}$ however, have not found letter alexia in alexia with agraphia, and indeed occasional cases of alexia with agraphia have been reported with intact oral spelling and comprehension of dictated, spelled words. ${ }^{28-30}$ Performance in letter versus word reading would thus not appear to be a reliable factor in distinguishing alexia with agraphia from alexia related to Wernicke's aphasia, though certainly it does distinguish alexia without agraphia, or "posterior alexia", from the alexia associated with frontal lesions. Inability to perceive a word as a whole is the most consistent deficit in "posterior" alexia, while single letter reading and phonetic processing of graphemes may be intact. In both the "anterior" and "central" alexias, on the other hand, whole word perception may be the only preserved function, while letter reading and phonetic processing are impaired. ${ }^{19} 2526$

Cases of Wernicke's aphasia with alexia out of proportion to auditory comprehension deficit have not been generally recognised, though as discussed above some cases of alexia with agraphia may belong to this category. Hécaen ${ }^{16}$ described a subset of Wernicke's aphasics with auditory predominant deficits, but he related this to a deficit in the perception of phonemes. His second subgroup, with deficient semantic comprehension, was said to have equal impairment of auditory comprehension and reading. Mohr, Hier, and Kirshner, ${ }^{10}$ in a retrospective review of CT scan lesions, found two cases of Wernicke's aphasia with greater impairment of reading than of auditory comprehension. Both had parieto-occipital lesions. This compared to six cases of auditory-predominant deficit and 23 in which no disproportion could be judged. The present three cases are part of an ongoing investigation of selective involvement of specific language modalities in aphasia secondary to ischaemic stroke. Of 18 cases of Wernicke's aphasia studied, two showed the auditory and three the visual-predominant syndrome. Selective impairment of either auditory comprehension or reading thus appears to be not uncommon in Wernicke's aphasia.

The independence of deficits in reading and auditory comprehension in Wernicke's aphasia suggests at least partial independence of the brain mechanisms for auditory and visual language processing. This independence belies the simple concept that reading can proceed only via transcoding of visual to phonetic information. The evidence 
from Wernicke's aphasia thus confirms evidence from other sources ${ }^{31}{ }^{32}$ that reading can occur from purely visual to semantic processing, without access to auditory or phonetic information.

This research was supported by NINCDS Teacher Investigator Development Award No. 5K07NS00429-03 to Dr Kirshner and also by the Veterans Administration. The authors thank Mrs Anne Morgan for technical assistance and Mrs Jane $S$ Smith for assistance with preparation of the manuscript.

\section{References}

${ }^{1}$ Benson DF, Geschwind N. The aphasias and related disturbances. In: Baker AB, Baker LH eds; Clinical Neurology. Hagerstown, Harper and Row Publishers, 1977, Vol 1, Chapter 8, 3-9.

${ }^{2}$ Brown J. Aphasia, Apraxia and Agnosia. Clinical and Theoretical Aspects. Springfield: Charles C. Thomas, 1972, 56-71.

${ }^{3}$ Benson DF. Aphasia, Alexia, and Agraphia. New York: Churchill Livingstone, 1979, 117.

${ }^{4}$ Kirshner HS, Webb WG, Duncan GW. Word Deafness in Wernicke's Aphasia. J Neurol Neurosurg Psychiatry, 1981;44:197-201.

s Sidman M, Stoddard LY, Mohr JP et al. Behavioral studies of aphasia. Methods of investigation and analysis. Neuropsychologia 1971;9:119-40.

- Goodglass H, Kaplan E. The Assessment of Aphasia and Related Disturbances. Philadelphia: Lea and Febiger, 1972.

${ }^{7}$ Spreen O, Benton A. Token Test. From the Neurosensory Center Comprehensive Examination for Aphasia, Neuropsychiatry Laboratory, University of Victoria, 1969.

${ }^{8}$ Gado M, Hanaway J, Frank R. Functional anatomy of the cerebral cortex by computed tomography. J Comput Assist Tomogr 1979;3:1-19.

${ }^{9}$ Wernicke C. The symptom complex of aphasia. A psychological study on an anatomical basis. Proc Boston Colloquium for Philosophy of Science 1968;4:34-97 (originally 1874).

${ }^{10}$ Mohr JP, Hier D, Kirshner HS. Modality bias in Wernicke's aphasia. Neurology (Minneap) 1978;28:395 (Abst).

"Heilman KM, Rothi L, Campanella D et al. Wernicke's and global aphasia without alexia. Arch Neurol 1979;36:129-33.

12 Stoddard WE, Davis DO, Young SW. Cortical blindness after cerebral angiography. Case report, J Neurosurg 1981;54:240-4.
${ }^{13}$ Bogen JE, Bogen GM. Wernicke's region-Where is it? Ann NY Acad Sci 1976;280:834-843.

${ }^{14}$ Dejerine MJ. Contribution à létude anatomopathologique et clinique des differenctes variêtés de cécité verbale. CR Soc Biol (Paris) 1892;4:61-90.

${ }^{15}$ Geschwind N. Disconnexion syndromes in animals and man. Brain 1965;88:237-94, 585-644.

${ }^{16}$ Hécaen H, Kremin H. Neurolinguistic research on reading disorders resulting from left hemisphere lesions: aphasic and "pure" alexias. In: Whitaker $\mathrm{H}$, Whitaker H eds; Studies in Neurolinguistics, Vol 2, New York; Academic Press, 1977.

${ }^{17}$ Kertesz A. Aphasia and Associated Disorders. Taxonomy, Localization, and Recovery, New York, Grune \& Stratton, 1979.

${ }^{18}$ Brain WR. Speech Disorders. London; Butterworths, 1962.

${ }^{19}$ Benson DF. The third alexia. Arch Neurol 1977; 34:327-31.

${ }^{20}$ Benson DF, Sheramata WA, Bouchard R, et al. Conduction aphasia. A clinicopathological study. Arch Neurol 1973;28:339-46.

${ }^{21}$ Warrington EK, Rabin P. Visual span of apprehension in patients with unilateral cerebral lesions. $Q J \operatorname{Exp}$ Psychol 1971;23:423-31.

${ }^{22}$ Staller J, Buchanan D, Singer M, et al. Alexia without agraphia: An experimental case study. Brain Lang 1978;5:378-87.

${ }^{23}$ Levine DN, Calvanio R. A Study of the visual defect in verbal alexia-simultanagnosia. Brain 1978;101:6581.

${ }^{24}$ Kirshner HS, Staller J, Webb WG, Sachs P. Transtentorial herniation with posterior cerebral artery territory infarction: a new mechanism of the syndrome of alexia without agraphia. Stroke, 1982;13:243-6.

${ }^{25}$ Benson DF, Brown J, Tomlinson EB. Varieties of alexia Word and letter blindness. Neurology (Minneap) 1971;21:951-7.

${ }^{26}$ Kirshner HS, Webb WG. Word and letter reading and the mechanism of the third alexia. Arch Neurol 1982;39:84-7.

${ }^{27}$ Alajouanine T, Lhermitte F, Ribaucourt-Ducarne B Les alexies agnostiques et aphasiques. In: Alajouanine $\mathrm{T}$ ed, Les grandes activités du lobe occipital. Paris, Masson, 1960.

${ }^{28}$ Albert ML, Yamadori A, Gardner $\mathrm{H}$ et al. Comprehension in alexia. Brain 1973;96:317-28.

${ }^{29}$ Mohr JP. An unusual case of dyslexia without dysgraphia. Brain Lang 1976;3:324-34.

${ }^{30}$ Rothi LJ, Heilman KM. Alexia and agraphia with spared spelling and letter recognition abilities. Brain Lang 1981;12:1-13.

${ }^{31}$ Kapur N, Perl NT. Recognition reading in paralexia. Cortex 1978;14:439-43.

${ }^{32}$ Saffran EM, Marin OSM. Reading without phonology: evidence from aphasia. $Q J$ Exp Psychol 1977;29:515-25. 